\title{
Digitalization in Hot and Cold Rolling Mills
}

\author{
Joachim Ohlert ${ }^{1, a^{*}}$, August Sprock ${ }^{1, b}$, Peter Sudau ${ }^{1, c}$ \\ ${ }^{1}$ SMS group $\mathrm{GmbH}$, Wiesenstraße 30, 57271 Hilchenbach \\ ajoachim.ohlert@sms-group.com, baugust.sprock@sms-group.com, \\ cpeter.sudau@sms-group.com
}

\begin{abstract}
Keywords: Data digging, intelligent component, assistance systems, autonomous process, coupling of autonomous processes
\end{abstract}

\begin{abstract}
The demands made by the market on the steel industry will again become significantly greater in the near future.

Examples of this are the expansion of the product mix, the production of smaller batch sizes, higher quality requirements, and lower prices. The paper presents possible responses of the equipment builder to these challenges. Digitalisation concepts should in particular support production engineering (hot/cold rolling) to the effect that new, mostly high-strength materials can be produced in an economically efficient way to hence allow their speedy introduction on the market.
\end{abstract}

\section{Introduction}

The demands made by the market $[2,3]$ on the steel industry will again become significantly greater in the near future. Detailed examples of this are:

$\rightarrow$ Expansion of the product mix in view of the prevailing competition with other materials (aluminum, CFRP), increasing variety of materials to be produced

$\rightarrow$ Need for faster adaptation to the current market requirements and thus shorter periods for the introduction of new or modified materials

$\rightarrow$ Production of smaller batch sizes with short delivery times

$\rightarrow$ Higher quality requirements (geometry, surface, microstructure)

$\rightarrow$ Increasing requirements for sampling and documentation during the production process and for product-related references and certificates

Lower prices, which have the effect of reducing the extent of maintenance, sampling and other quality-assuring measures

Besides the development of the materials, which is the primary task of the steel industry, the plantmaker must provide improved mechanical components and innovatory technologies and tools to enable rapid and reliable tests to be performed on the production of new steel grades, so that these can then be manufactured permanently on an industrial scale, with high productivity and uniform quality.

On the basis of the above, the essential requirements made on plantmakers $[2,3]$ are:

$\rightarrow$ Increased efficiency of plants, processes and products

$\rightarrow$ Effective processes (e.g. availability, yield)

$\rightarrow$ Resource-saving processes (energy, water, alloying elements, etc.)

$\rightarrow$ Intelligent process control systems

Efficiency includes the service life and safety of the plant components, the power density and overall power of the plants and products, and the product quality and process reliability. A prerequisite for fulfilling the requirements is the utilization of suitable mechanical equipment and technology. 
Intelligent process control systems represent the enhancement of the conventional process model technology (calculation of rolling forces, roll gaps, CVC positions, water flow rates) with a view to obtaining assistance systems intended to support the operator by means of optional proposals for action and/or automatic intervention in the process.

The aim is to ensure that the respective production processes, in this case hot rolling and cold rolling, function in a stable manner in spite of variable entry conditions of the input product, the process or the mechanical equipment and that the mainly narrow product tolerances are attained reliably and lastingly. Assistance systems for the operators simplify demanding and critical procedures, such as threading during the hot and cold rolling of high-strength materials.

\section{The "Intelligent Hot and Cold Rolling Mill"}

To enable the requirements described above to be fulfilled, today's conventional manufacturing process will in future be replaced by fabrication in the new "Intelligent hot and cold rolling mill" [2, $3,4]$.

The major core elements of the "Intelligent hot and cold rolling mill" are:

- The "Intelligent machine" and the "Intelligent machine element"

- The assistance system

- The "Autonomous process"

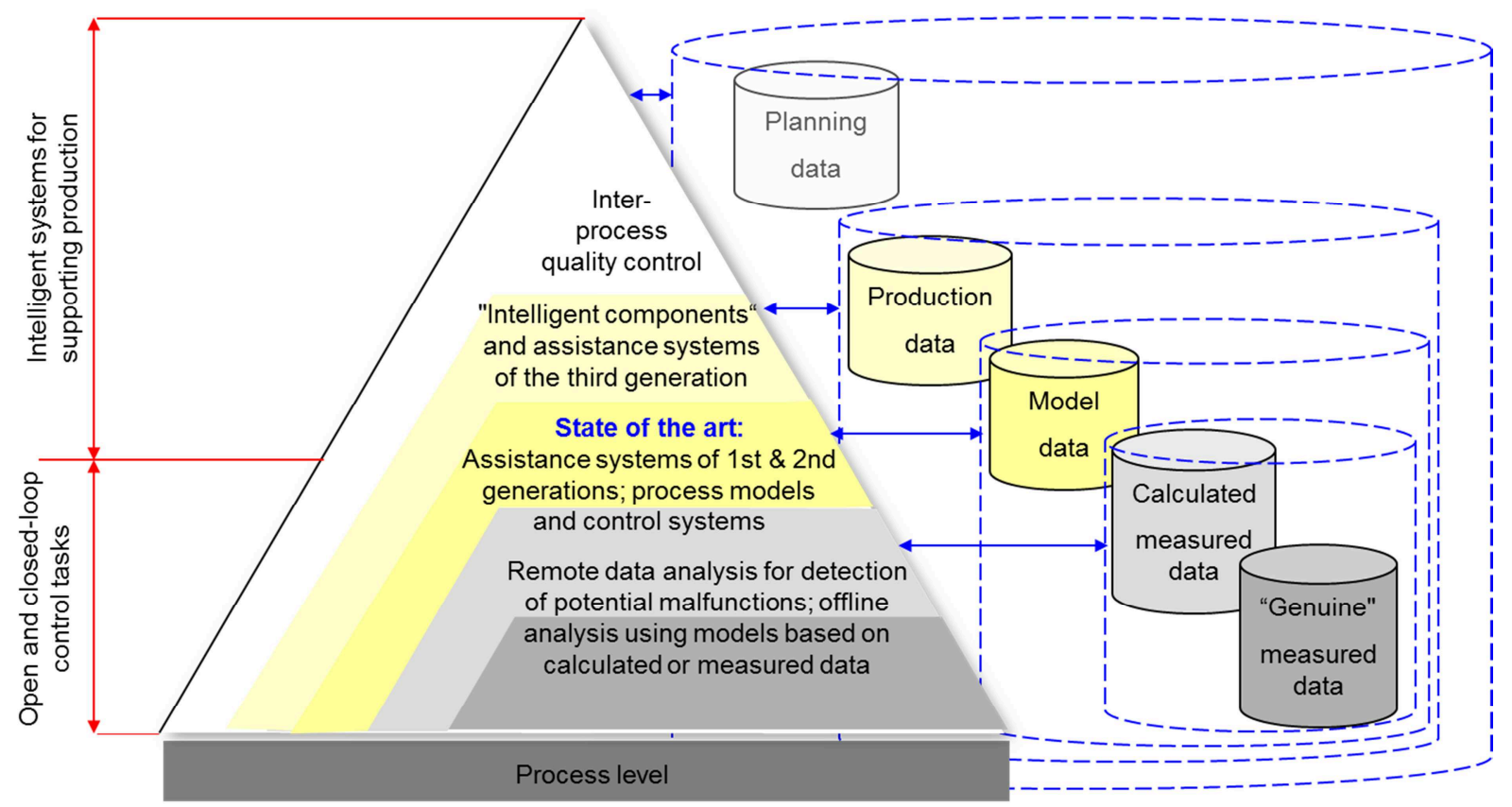

Fig. 1: Tasks of the various automation levels

Fig. 1 shows the integration of the future functions into the various automation levels. According to the current state of the art, process models and control systems deal primarily with the setup and control of the rolling mill (e.g. roll gaps and motor speeds) and the ensuing quality of the rolled stock [1]. The complexity of the hot rolling and cold rolling processes will continue to increase thanks to the growing demands, often contradictory in nature, made by customers on processes and products, such as flexibility and response speed on the one hand and consistency, uniformity, reproducibility and effectiveness on the other hand. This is particularly true as regards the rolling of new products (ultra-high-strength steels) which react considerably more sensitively to process malfunctions than do conventional steel grades. With the aid of the innovatory functions, which are mainly assigned to the second-highest level, the rolling process nevertheless remains stable and the required properties are reliably achieved. This is because the process transparency is enhanced and the current state of the mechanical equipment, process and input product is recognized and taken 
into account at all times (e.g. early, also preventive, diagnosis of malfunctions of the mechanical components).

The highest process level deals with global subjects such as increase of yield for the overall process, maintenance and optimization of quality, and also saving of resources (above all energy and alloying elements).

The availability of suitable measured values is of crucial importance for achieving the process transparency described above and for recording the condition of mechanical equipment, processes and products at a given moment. Modern hot and cold rolling mills are therefore equipped with 2000 to 10,000 measuring channels. Some of the individual measuring channels are scanned at a rate of 1 to 2 milliseconds. These channels, needed for the process and the monitoring, comprise both actual measurements, such as thickness and flatness, and calculated measurement variables (known as soft sensors) such as thermal crown and the roll wear.

The quality of the measured values and thus also the analysis of this quality are extremely important for all functions described so far and still to be described. In many measuring devices, a defect can be recognized by the operator only by means of a "healthy" signal or an implausible signal curve. However, the "healthy" signal only verifies a functionally reliable condition of the measuring device and does not say anything about the quality of the measurement itself, whereas the interpretation of the signal curve depends on the operator concerned and is therefore subjective to a very high degree. Faults affecting a measurement, such as those caused by water or steam, are therefore often recognized and eliminated only after several hours or days have elapsed. Measurements of temperature and width are particularly vulnerable to this. Today, since the process models rely heavily on correct measured values in order to guarantee sufficient precision for the rolling mill setup and a stable rolling process, they themselves already independently evaluate quality parameters of the measuring segments (e.g. standard deviation) and mutually correlate the measured values from several measuring devices in order to automatically exclude faulty measurements from the adaptation and other important functions.

The "threesome" comprising availability, ability to evaluate and accuracy of data will continue in the future to retain its strong significance. Furthermore, competence in making data from various sources and in various forms available on a common basis and interlinking these data in a suitable manner will become increasingly important.

\section{The "Intelligent Machine" and the "Intelligent Machine Element"}

An "Intelligent Machine" has direct access, on the element itself and thus on a decentralized basis, to all important data relating to manufacture and operation, to control data and calculated parameters, which can then be called up and used by various groups (e.g. control personnel, maintenance personnel, systems engineers). Advantages of a decentralized, self-contained solution are:

- Information on the current condition is directly available locally.

- By means of analyzing the information, it is possible to evaluate the load on the component and the further procedure can be derived from this.

- Operation is simple and the transparency is high because the respective data required for the various user groups can be provided in a targeted manner (data compression).

- The decentralized solution can be applied universally for all automation systems.

It is thus possible, depending on the operating conditions and state of the machine elements,

- to either reduce the power and load or to increase them

- to initiate necessary maintenance or to postpone it

- to operate the plant at the power limit without an increased risk

- to specify variable, condition-dependent maintenance intervals, which will ensure that the maintenance and repair costs can be reduced. 


\section{Local rotating system}



Fig. 2: Schematic diagram of a self-contained local system, using the example of a gear spindle

A computer system adapted to the requirements, comprising a CPU, measured-value acquisition and data memory, is integrated for the self-contained component represented in Fig. 2. This system makes it possible to record, compress, evaluate and store measured data. In addition, model-based calculations can be performed and, with Bluetooth LE for example, be transferred to an external system.

Various computer prototypes for the "Intelligent Machine" are currently being examined for their service lives under extreme loads, e.g. those arising during hot rolling. The different prototypes vary with regard to the sensor technology as adapted to the respective task, to the scanning rate (e.g. dependent on the process event) and to the analyses performed on the "Intelligent Component". Here, the energy consumption of the overall system plays just as essential a part as does the requirement for storage space. Since May 2015 a prototype has been in operation on a reference plant, and the results are currently being analyzed. The pilot series type is to go into operation in mid- 2016 on the basis of this analysis.

The energy required for operation of the processor and of the measuring sensors is to be generated not by batteries but with the aid of "energy harvesting". The theoretical examinations have been carried out and a prototype is being planned.

Fig. 3 shows a diagram of a model with optimized calculation speed, which computes the current condition of the teeth of a gear spindle from measured operating data. The control variable for the simulation is the calculated temperature, which is compared with the measured temperature (cf. Fig. $3)$. The results are used for specifying the maintenance intervals.

Therefore, the "Intelligent Machine" can, on the one hand, communicate with the outside world and supply the information needed at a given moment and, on the other hand, it possesses a "component memory" in which its history is stored. This history contains all stages of the manufacturing process and the various stages of operation. Thus, for example, a machine element that is currently in the repair shop knows which status of its life-cycle it has reached.

If an "Intelligent Machine" forwards its information to the "mechanical assistant", then this assistant can decide, for example, whether a machine element such as a roll continues to be suitable for rolling schedules with high loads or whether it can only be used within the range of low or medium stresses. This leads to improved utilization of the operating materials and to reduction of outage times that may be caused by component failure and in some cases result in high costs. 
The transition to assistance systems of the more recent generation is represented by the interactive and dynamic utilization of the information available on the machine element. With the aid of the calculation results obtained there, the rolling process can be varied and optimized, the rolling mill controlled and the efficiency of the complete plant increased. To do this, however, suitable higher-level models must be available, which can coordinate the individual "Intelligent Machines" and prioritize adequately with regard to the overall process.



Fig. 3: Coupling of operating data for mechatronic models

As described, the use of "Intelligent Machines" allows outage times to be reduced and operating materials to be saved. In view of the immense pressure of costs to which the steel industry is at present being subjected and which has resulted in the continual limitation of predictive maintenance measures, the provision of support to process engineers and maintenance personnel via suitable intelligent systems does furthermore appear to be more or less the only possibility in the future for assuring the reliable and reproducible operation of the plant on a lasting basis.

\section{Assistance Systems of the First Generation}

From around the year 2003, the first assistance systems were developed and put into operation. They are based primarily on the skillful analysis of large quantities of data, on the recognition of interrelationships and patterns and on the synthesis of suitable instructions for action. The assistance systems of the first generation do not provide for any automatic interventions in the process. The subject matter and facts examined are of limited complexity since the millstands in the rolling mill are considered individually and in isolation from each other. The assistance systems provide the operator with support and help with decision-making. A typical representative of the first generation of assistance systems is the tail out monitor or tail out assistant in hot rolling. It assists the operator when levelling the roll gap for the rolling of strip tail-ends, in order to prevent runout of the strip and the consequent damage to rolls and rolled stock in the form of surface marks.

In the tail out assistant, various signals such as differential rolling force are automatically analyzed and, on the basis of the calculated results, the operator is supplied with levelling recommendations for the next strip. Orange color for example signifies that no damage to the strip tail-ends has as yet occurred but that it is necessary for the operator to intervene and perform 
correction of the levelling amount by approx. $20 \mu \mathrm{m}$ to $40 \mu \mathrm{m}$. The levelling direction is indicated by an arrow. In combination with the modernization of automation systems, the introduction of this assistance system has significantly reduced the number of interruptions of rolling and rolling aborts and has increased the productivity by up to $50 \%$, particularly in the rolling of high-strength steel grades.

In addition to the Level 1 functions described, the assistance systems of the first generation include systems that are assignable to Level 2. In principle, they possess the same structure.

Examples:

- Early analysis of rollability

- Preparation of an alternative pass schedule for cases where the temperature is not attained

Here also, automatically and with incorporation of the customer's available primary data, options for action are generated and suggested to the operator. If the operator accepts a suggestion from the assistant, the suggestion is implemented by the Level 2 system and the rolling process is adapted accordingly.

\section{Assistance Systems of the Second Generation}

The complexity was increased in the assistance systems of the second generation. At the same time, automatic interventions in the process were allowed. In the threading assistant for tandem cold mills, the roll gaps are corrected according to specified rules before threading-in, depending on the quality fluctuations of the previous stages (e.g. thickness tolerances of the hot strip, machining status of the product, etc.), and operating mode of the automatic control systems is adapted. Besides reliable threading-in and thus the increasing of productivity, the purpose of the assistance system is to minimize the off-gage lengths upon start of rolling, especially in the case of multiple rolling operations without intermediate annealing. The ensuring of good strip flatness by correspondingly setting the flatness actuators is a prerequisite for utilizing this assistance system.



Fig. 4: Ramp-up curve for new automation using the threading assistant

In Fig. 4 the results are shown for the threading assistant, using the example of the ramp-up curve of the tandem cold mill for medium-wide strip at Bilstein following the revamping of the automation system. Bilstein is a producer of high-strength and ultra-high strength steels. The cold mill processes materials with an entry yield point of $\geq 1200 \mathrm{~N} / \mathrm{mm}^{2}$. In the meantime, thanks to the 
new automation system and the assistance systems, the productivity has been increased by approx. $20 \%$.

The Level 1 assistance systems of the second generation are completed by corresponding Level 2 assistants. These include:

- Generation of adaptation values for new products

- Thread-in optimization

- Redistribution of roll force

- Replacement strategies in case of data failure

\section{Assistance Systems of the Third Generation}

The assistance systems of the third generation are at present both in the development phase and in the prototype phase. The systems are different from those of the second generation, particularly with regard to their (once again heightened) complexity and the (greatly expanded) functions. Their purpose is to automatically analyze interrelationships (e.g. deviation of the mechanical load from the desired condition) and to prepare countermeasures, which must be either carried out manually (repair of defective components, such as spray nozzles on the descaler) or can be performed automatically (process alterations for reducing the load). An overview of the most important modules is shown in Fig. 5. The functions of the assistance systems are briefly explained below:

Basis: $\quad$ Reliable acquisition and storage of process and production data for all important subprocesses (horizontal production chain) and automation levels (vertical structure of the automation pyramid)

Tasks: Automatic compilation, visualization and analysis of the data; feedback of findings in the form of instructions for action to the process computer system and to the personnel

\footnotetext{
Aims: - Increasing of process transparency

- Improvement of process stability, thus ensuring uniform quality

- Prevention of product downgrading by means of timely intervention in the production sequence

- $\quad$ Enhancing of plant availability

- Detection of causes of faults and taking of relevant countermeasures

- Supporting and simplifying of complex analysis steps, thus saving time

- Improvement of operability and ease of handling
} 


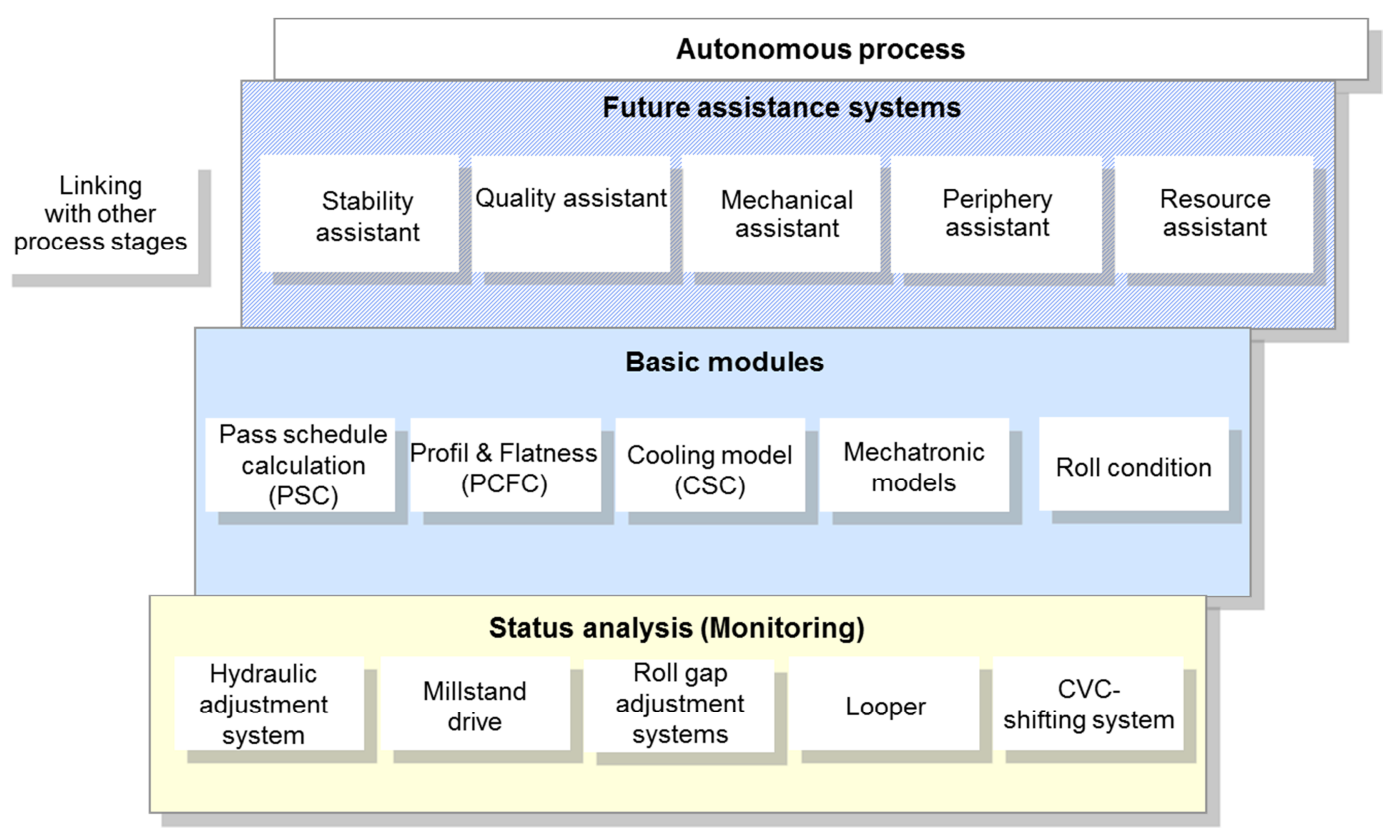

Fig. 5: Assistance systems of the third generation (extract)

A quality assistant module that is in the prototype phase is, for example, the Microstructure Property Model (cf. Fig. 6) [5, 6, 7] which, like most models of SMS group, can be operated both online and offline. The major online functions are briefly described as follows.

Observation Mode. The existing production is tracked and the impact is calculated of the thermal and mechanical load on the microstructure and on the mechanical properties during rolling and cooling. With the aid of this module, the Quality Control department can, for example, decide very quickly whether a strip can be released for acceptance in cases where the process parameters (e.g. rolling temperatures, cooling speci-fications) have not been observed.

Intervention in the Process Conditions. In the event of the process parameters changing in the previous processing steps, the coiler temperature can be newly determined and freely controlled during operation, in order to attain the intended properties.

Furthermore, the following offline functions are possible:

Simulator Mode. This mode can be utilized for the development of materials. Prediction can be made of the dimensions (width, thickness) in which a material with altered chemical composition can be manufactured without exceeding the capacity limits of the rolling mill (torque, roll force). After the parameters for rolling and cooling have been specified, the material is manufactured and sampled in the rolling mill. The development period for new materials can be significantly shortened by using the Microstructure Property Model. 


\begin{tabular}{|l|}
\hline Input variables \\
\hline Hot rolling mill data \\
\hline Hot strip data \\
\hline \begin{tabular}{l} 
Reheating, rolling and \\
cooling conditions \\
- Furnace temperature \\
- Rolling temperatures \\
- Coiling temperature \\
- Pass schedule \\
- Cooling strategy \\
\hline
\end{tabular} \\
\hline
\end{tabular}
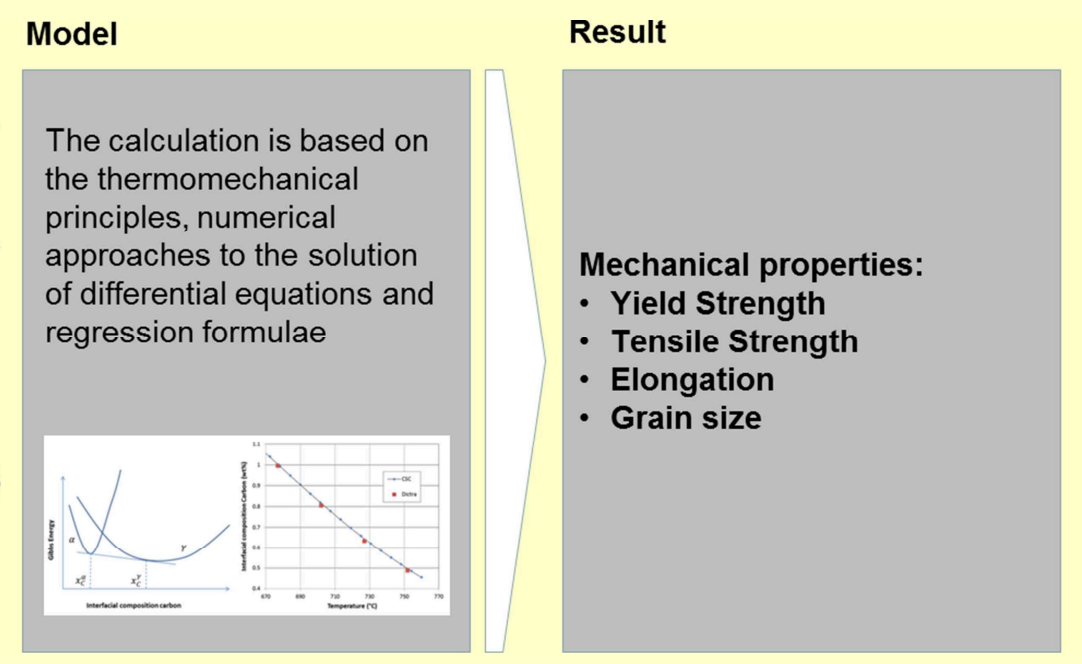

Fig. 6: Basic structure of the Microstructure Property Model

Optimization of the Alloying Concepts for an Existing Product Mix. The influence of the cooling rate on the properties can be determined in quantitative terms by examining the interrelationships arising from plant, process and material technology. It is thus possible to adapt existing chemical compositions in respect of the attainable strength values or to modify them by making use, for example, of fast cooling in order to save on alloying elements.

Fig. 7 displays operating results before and after the revamping of a cooling section. The maximum possible cooling rate has been doubled and, in addition, the Microstructure Property Model has been implemented. The results measured and displayed in the diagram (red squares) show that the tensile strength of various materials (HSLA and CMn grades) was able to be increased by an average of up to $15 \mathrm{MPa}$, while the elongation remained constant, as had been predicted by the model. The calculated data are shown with the green triangles and are in accordance with the measured data (red squares). This makes it possible for the mill owner to produce higher-strength materials or to reduce the content of alloying elements if the strength is to remain unchanged.



Fig. 7: Operating results of the Microstructure Property Model on a revamped cooling section 


\section{Future Prospects: Achievement of an "Autonomous Process" via the Interlinking of Various Intelligent Functions}

It is intended that in the future the combination of comprehensive data analysis, today's basic modules for automation and assistance systems of varying degrees of complexity will make it possible, at an early stage, to prepare the operator or the model to make a decision as to whether to intervene in the subsequent process stages so that by means of targeted process alterations the customer's specifications can be reliably attained, or even improved upon, in spite of deviations occurring during the previous process stages. The customer's specifications here are understood to be the clearly specified product characteristics and quality parameters such as geometry and mechanical properties and, over and above this, also global production objectives such as increased yield and the minimizing of the operating materials used and, in particular, energy. As already mentioned, the highest process level is responsible for the achievement of these overall objectives.

On a similar plane to the current discussion about the "autonomous driving" of modern motor vehicles is the need to clarify the question as to what role the operating personnel are to adopt in the future. Here, questions of responsibility and liability play just as important a part as do those relating to availability and protection of know-how. In the final analysis, these topics directly or indirectly concern the owner's philosophy.

Independently of this, the "Autonomous Process" should make it possible in the future, on the basis of a real-time analysis of the current product quality, to provide suggestions for the still outstanding, downstream process stages and thereby safeguard the quality of the final product. Such suggestions may well vary, depending on the customer's overall aims. Thus, various modes of procedure are necessary and conceivable with a view to maximizing production, minimizing consumption rates or manufacturing special, advanced-technology products.

If, for example, the analysis deviates from the desired value in the steel plant, the hot rolling strategy must be adapted so as to enable the recrystallization behavior and thus the strip steering to remain stable. The Microstructure Property Model additionally calculates a suitable cooling strategy, in accordance with the process adaptations, in order to safeguard the achievement of the required material properties. If the available possibilities for intervention do not enable the quality of the final product to be ensured, then a decision can be made at an early point in time, by the operator or automatically by the model, as to whether the desired values for the product properties should be modified or whether the manufacturing process should be aborted at this point. Consequently, transparent documentation of the current condition of the process and product represents the basis for the ability to introduce autonomous processes.

\section{References}

[1] D. Rosenthal, P. Sudau, New technologies for manufacture of thin high-strength hot and cold strip, Aachen Steel Colloquium 2004

[2] Dieter Spath, Fraunhofer IAO, internal lecture, Innovation aspects in machine and equipment building, with special consideration to process-oriented services

[3] Process-oriented services work carried out at various customers

[4] Prof. Dr.-Ing. Volker Stich, internal Industry 4.0 Management-Workshop

[5] Herman, J.C., B. Donnay, A. Schmitz, U. Lotter, R. Grossterlinden, Computer assisted modelling of metallurgical aspects of hot deformation and transformation of steels, Technical steel research, Final Report EUR 18790

[6] Herman, J.C., B. Thomas, U. Lotter, Computer assisted modelling of metallurgical aspects of hot deformation and transformation of steels, Technical steel research, Final Report EUR 16795

[7] Hodgson, P.D., R.K. Gibbs, A mathematical model to predict the mechanical properties of hot rolled C-Mn and microalloyed steels, ISIJ Intern. 32, 1992, no. 12, pp. 1329 - 1338 